\title{
Seguridad y mayor tolerancia al esfuerzo con entrenamiento interválico en comparación con el entrenamiento de intensidad moderada continua en cardiópatas de riesgo cardiovascular muy alto
}

\section{Safety and improvement in exercise tolerance with interval training vs moderate-intensity continuous training in heart disease patient of very high cardiovascular risk}

\author{
Alfredo D. Pineda-García ${ }^{1 *}$, Jorge A. Lara-Vargas ${ }^{1}$, Andrés Ku-González ${ }^{1}$, Víctor J. Lastra-Silva1, \\ Rodolfo Arteaga ${ }^{1}$ y Juan A. Pineda-Juárez ${ }^{2}$ \\ ${ }^{1}$ Servicio de Rehabilitación Cardíaca, División de Servicios Modulares, Facultad Mexicana de Medicina, Universidad La Salle; ${ }^{2}$ Departamento de \\ Investigación Clínica. Centro Médico Nacional 20 de Noviembre, Instituto de Seguridad y Servicios Sociales de los Trabajadores del Estado, Ciudad \\ de México, México
}

\begin{abstract}
Resumen
Objetivo: Evaluar la seguridad y el efecto del entrenamiento por intervalos de moderada y alta intensidad (EIMI, EIAl) y compararlo con el entrenamiento de intensidad moderada continua (EIMC) en pacientes de riesgo cardiovascular (RCV) muy alto. Método: Estudio cuasi experimental de 81 pacientes que ingresaron al programas de rehabilitación cardíaca y prevención secundaria (PRHCPS); en la estratificación de riesgo, todos los pacientes presentaron $\geq 2$ factores de RCV alto. Entrenamiento concurrente: aeróbico supervisado: 2 semanas de entrenamiento de base de EIMC; luego el grupo EIMC con la misma prescripción, EIMI a 3 intervalos de 3' al 70 a $80 \%$ de FCR con pausas activas de 3' al 60 a $70 \%$ de FCR; EIAl, 3 intervalos de 3' a 80 a $90 \%$ de FCR con pausas activas de 3' al 70 a 80\% de FCR, entrenamiento de fuerza con tres sesiones semanales de ejercicios isotónicos y una de calistenia e intervención multidisciplinaria. Resultados: 81 pacientes asignados en tres grupos: 61 a EIMC, 13 a EIMI y 7 a EIAI, sin diferencias significativas en las variables epidemiológicas. Hubo ganancias porcentuales en MET-carga de $45 \%, 60 \%$ y $86 \%$ ( $p=0.17)$ y carga-vatios de 51, 44 y 48 en EIMC, EIMI, EIAI, respectivamente $(p=0.54)$. A pesar de no existir diferencias estocásticamente significativas intergrupales, sí se registró una ganancia mayor en MET-carga en el grupo de EIAI. No se observó desenlace adverso en ningún tipo de entrenamiento. Conclusiones: En cardiópatas de riesgo cardiovascular muy alto, el entrenamiento interválico EIMI/EIAl supervisado es seguro, con tendencia a mayor ganancia en tolerancia al esfuerzo (MET-carga) con el EIAl en comparación con el EIMC.
\end{abstract}

Palabras clave: EIMC. EIMI. EIAI. Alto riesgo.

\begin{abstract}
Objective: Evaluate safety and effects of training at moderate and high intensity intervals (MIIT, HIIT) compared to continuous moderate intensity training (MICT) in heart disease patient with very high cardiovascular risk (CVR). Method: Quasi-experimental study of 81 patients in a cardiac rehabilitation (CR) program, after risk stratification, all patients presented at least 2 factors with

Correspondencia:

*Alfredo D. Pineda-García

E-mail: pinedacardio@gmail.com CC BY-NC-ND (http://creativecommons.org/licenses/by-nc-nd/4.0/).

Fecha de recepción: 15-02-2020

Fecha de aceptación: 22-04-2020 DOI: 10.24875/ACM.20000071

Disponible en internet: 12-04-2021 Arch Cardiol Mex. 2021;91(2):178-185 www.archivoscardiologia.com
\end{abstract}


high CVR. All patients were provided supervised concurrent training: aerobic: initially 2 weeks MICT. subsequently MICT group with same prescription, MIIT 3 intervals of 3 'to 70-80\% FCR with active pause exercise of 3' to 60 a 70\% FCR, HIIT 3 intervals of 3'a $80-90 \%$ FCR with active pause exercise of $3{ }^{\prime} 70$ a $80 \%$ FCR, resistance training 3 weekly sessions isotonic exercises and 1 calisthenics and multidisciplinary intervention. Results: 81 patients assigned to: 61 MICT group, 13 MIIT and 7 HIIT, no significant differences were observed. Percentage gains were obtained in METs-load $45 \%, 60 \%$ and $86 \%(p=0.17)$ and watts 51,44 and 48 in MICT, MIIT, HIIT respectively $(p=0.54)$. Although there are no statistical significant intergroup differences if there is a greater improvement in MET-load in the HIIT group. There was no adverse outcome in any training. Conclusions: In very high cardiovascular risk heart disease patient, supervised interval training (MIIT/HIIT) is safe, with a tendency to greater improvement in exercise tolerance (METs-load) with HIIT compared to MICT.

Key words: MICT. MIIT. HIIT. Cardiovascular risk.

\section{Introducción}

La enfermedad cardiovascular (ECV) es hoy día la principal causa de mortalidad en el mundo, México incluido. Los programas de rehabilitación cardíaca y prevención secundaria (PRHCPS) han demostrado ser efectivos en la reducción de la mortalidad cardiovascular y por todas las causas, la disminución de la reincidencia de infarto y la mejoría de la calidad de vida; dichos resultados son dependientes de la dosis ${ }^{1-3}$. La prescripción del ejercicio y la gradación del riesgo cardiovascular son los pilares de la ejecución correcta de la intervención ${ }^{4}$.

Por lo regular, el entrenamiento de intensidad moderada continua (EIMC) ha sido la base del entrenamiento en los programas de rehabilitación cardíaca y prevención secundaria (PRHCPS). Sin embargo, en evidencia más reciente, el entrenamiento interválico de moderada intensidad (EIMI) y el entrenamiento interválico de alta intensidad (EIAI) han demostrado ser seguros y obtener mejores resultados en ganancia en el consumo máximo de oxígeno (VO2p) en pacientes estratificados como riesgo cardiovascular (RCV) bajo a moderado, en comparación con el EIMC. Dicha evidencia está sustentada en el hecho de que el desplazamiento de umbrales mayores (VT2 y VO2p) en deportistas sanos puede replicarse en pacientes con cardiopatía al margen de su gradación del riesgo $0^{1-3,5}$. Aunado a ello, las características de la población mexicana comparten múltiples variables de alto riesgo cardiovascular y los pacientes se catalogan como de RCV "muy alto".

Hasta el momento se desconocen los efectos en seguridad y ganancia en tolerancia al esfuerzo del EIMI/EIAI en comparación con el EIMC en enfermos cardiópatas de RCV muy alto. La hipótesis de los autores establece que si las intervenciones de mayor intensidad (a tolerancia interválica) generan mayor volumen de entrenamiento, y dado que éste se correlaciona directamente con ganancias mayores en VO2p y resultados angiogénicos, regulación autonómica y endotelial a largo plazo ${ }^{6-7}$, sin sufrir complicaciones mayores, entonces los pacientes de riesgo cardiovascular muy alto podrían obtener resultados mayores con EIAI/ EIMI en términos de eficiencia y seguridad que aquéllos sometidos a EIMC. El objetivo de este estudio es evaluar la seguridad y los efectos del entrenamiento interválico (EIMI/EIAI) comparados con el entrenamiento de intensidad moderada continua en pacientes de riesgo cardiovascular muy alto.

\section{Material y métodos}

Estudio cuasi experimental de 81 pacientes que ingresaron al PRHCPS, previa estratificación del RCV mediante prueba de ejercicio; todos los pacientes tenían al menos dos factores de RCV alto (tabla 1). Todos los individuos se sometieron a entrenamiento aeróbico en cicloergómetro o banda sin fin de acuerdo con el principio de individualización del entrenamiento durante cuatro a seis semanas, cinco sesiones semanales de 30 minutos; todos los pacientes llevaron durante dos semanas entrenamiento base de EIMC, 5 min de calentamiento, $20 \mathrm{~min}$ al $70 \%$ de la frecuencia cardíaca de reserva (FCR) por fórmula de Karvonen, y 5 min de enfriamiento; según fueran la evolución, el criterio médico y el riesgo cardiovascular, los pacientes se categorizaron después para implementación de ElMI-EIAI. El grupo de EIMC tuvo la misma prescripción establecida; el grupo de EIMI, 5 min de calentamiento, tres intervalos de $3 \mathrm{~min}$ al 70 a $80 \%$ de FCR con pausas activas de 3 min al 60 a $70 \%$ de FCR, seguidos de 5 min de enfriamiento; y para el grupo de EIAI, $5 \mathrm{~min}$ de calentamiento, tres intervalos de $3 \mathrm{~min}$ al 80 a $90 \%$ de FCR con pausas activas de $3 \mathrm{~min}$ al 70 a $80 \%$ de FCR, y 5 min de enfriamiento (Fig. 1). Todos los pacientes recibieron tratamiento concurrente con entrenamiento de fuerza de tres sesiones semanales con 
Tabla 1. Factores de riesgo cardiovascular alto

\begin{tabular}{l} 
Paciente con síntomas $<5$ MET \\
Paciente con infarto de miocardio complicado \\
Tolerancia al esfuerzo $<5$ MET \\
Paciente con isquemia con descenso del ST $>2$ mm y FC $<135$ \\
Ipm \\
Respuesta hipotensora o plana \\
Superviviente de paro cardiorrespiratorio \\
Enfermedad coronaria ( $>50 \%$ ) grave o no revascularizable \\
FEVI < 40\%. Alteraciones de la motilidad mediante \\
ecocardiografía, acinesia o discinecia \\
Repuesta cronotrópica $<85 \%$ de la FC máxima \\
Recuperación < 12 Ipm al primer minuto \\
Respuesta presora $<1.23$ \\
Recuperación de la tensión arterial sistólica $>0.95$ \\
EVF esfuerzo y recuperación \\
Índice de DUKE <- 11 o veteranos $>2$ \\
Isquemia en estudio de medicina nuclear \\
Enfermedad arterial coronaria no revascularizable \\
Ectopia ventricular compleja: contracciones ventriculares \\
prematuras multifocales, taquicardia ventricular, fibrilación \\
ventricular \\
Presencia de depresión clínica \\
\hline
\end{tabular}

MET: tolerancia al esfuerzo; FC: frecuencia cardíaca; FEVI: fracción de expulsión del ventrículo izquierdo; EVF: extrasístoles ventriculares frecuentes; MN: medicina nuclear.

ejercicio isotónico y una sesión de calistenia, así como intervenciones grupales de nutrición y control de factores de riesgo cardiovascular, atención psicoemocional, terapia ocupacional, y técnicas antitabaco y sexualidad.

Todas las sesiones tuvieron supervisión de un cardiólogo rehabilitador y se realizó escrutinio de seguridad antes de las sesiones, las cuales se vigilaron mediante telemetría, con registro de presión arterial, antes, durante y después de las sesiones de ejercicio dinámico-estático. Para el análisis estadístico, los datos obtenidos se capturaron en una hoja de cálculo en el programa Microsoft Excel y se analizaron con el programa estadístico SPSS v22. Para las variables cuantitativas continuas, los resultados se presentaron en medianas y percentiles y como frecuencia y porcentajes cuando fueron categóricas. Para la comparación entre los grupos de estudio se utilizó la prueba de Kruskall-Wallis en

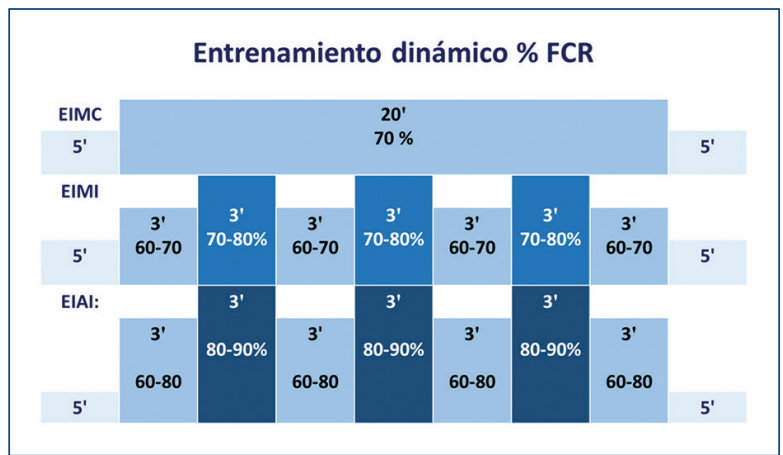

Figura 1. Entrenamiento por intervalos y entrenamiento moderado continuo. FCR: porcentaje de frecuencia cardíaca de reserva; EIMC: entrenamiento moderado continuo; EIMI: entrenamiento interválico de moderada intensidad; EIAI: entrenamiento interválico de alta intensidad. (autor: Alfredo D. Pineda García).

el caso de las variables continuas y $\chi^{2}$ de Pearson en el caso de las variables categóricas. Para la comparación entre los grupos de estudio después de la intervención se usó la prueba de Kruskall-Wallis.

\section{Resultados}

Se incluyó en el estudio a 81 pacientes distribuidos en tres grupos: grupo EIMC con 61 pacientes y promedio de edad de 65 años, con 44 del género masculino (72.1\%) y FEVI promedio de 52\%; grupo EIMI con 13 pacientes y promedio de edad de 70 años con 9 del género masculino $(62.3 \%)$ y $\mathrm{FEVI}$ promedio de $59 \%$; grupo EIAI con 7 pacientes y promedio de 66 años de edad, con 6 del género masculino (85.71\%) y FEVI promedio de $56 \%$, sin diferencia significativa $(p=0.03)$.

No se identificó diferencia significativa entre género $(\mathrm{p}$ $=0.80)$, edad $(p=0.36)$ o FEVI $(p=0.3)$. Se obtuvieron los factores de riesgo cardiovascular como obesidad, tabaquismo, diabetes mellitus tipo 2 (DM2), hipertensión arterial sistémica (HAS), dislipidemia y sexualidad, sin observar diferencias significativas entre las variables epidemiológicas (tabla 2). Los diagnósticos más prevalentes fueron cardiopatía isquémica seguida de insuficiencia cardíaca. Se obtuvieron ganancias porcentuales en MET-carga de $45 \%, 60 \%$ y $86 \%$ ( $p=0.17$ ) y carga-vatios de 51, 44 y 48 en EIMC, EIMI, EIAI, respectivamente $(p=0.54)$. A pesar de no existir diferencias estocásticamente significativas intergrupales, sí se reconoció una ganancia en MET-carga en el grupo EIAI (85.74\%, $p=$ 0.171). No hubo episodio cardiovascular adverso en ninguna de las sesiones de entrenamiento (tabla 3). 
Tabla 2. Características de la muestra

\begin{tabular}{|c|c|c|c|c|}
\hline Variable & EIMC $n=61$ & EIMI $n=13$ & EIAI n = 7 & $\mathbf{p}$ \\
\hline $\begin{array}{l}\text { Sexo, n (\%) } \\
\text { Mujer } \\
\text { Hombre }\end{array}$ & $\begin{array}{l}17(27.9 \%) \\
44(72.1 \%)\end{array}$ & $\begin{array}{l}4(30.76 \%) \\
9(69.23 \%)\end{array}$ & $\begin{array}{l}1(14.28 \%) \\
6(85.71 \%)\end{array}$ & 0.80 \\
\hline Edad (años) & $65(56.5-70)$ & $70(56.5-74.5)$ & $66(56-70)$ & 0.36 \\
\hline IMC & $27.73(25.39-30.52)$ & $28.71(24.89-30.88)$ & $26.18(23.45-28.95)$ & 0.61 \\
\hline Obesidad, n (\%) & $29(47.50 \%)$ & $8(61.50 \%)$ & $2(28.60 \%)$ & 0.446 \\
\hline Tabaquismo, n (\%) & $37(60.70 \%)$ & $6(46.20 \%)$ & $7(100 \%)$ & 0.52 \\
\hline $\mathrm{DM} 2, \mathrm{n}(\%)$ & $26(42.60 \%)$ & $5(38.50 \%)$ & $3(42.90 \%)$ & 1 \\
\hline HAS, n (\%) & $43(70.50 \%)$ & $10(76.90 \%)$ & $4(57.10 \%)$ & 0.65 \\
\hline Dislipidemia & $36(59.00 \%)$ & $10(76.90 \%)$ & $4(57.10 \%)$ & 0.537 \\
\hline Sexualidad (\%) & $29(47.50 \% \%)$ & $9169.20 \%$ & $4(57.10 \%)$ & 0.301 \\
\hline $\mathrm{Cl}, \mathrm{n}(\%)$ & $54(88.50 \%)$ & $11(84.50 \%)$ & $7(100 \%)$ & 0.70 \\
\hline IC, n (\%) & $23(37.70 \%)$ & $3(23.10 \%)$ & $1(14.30 \%)$ & 0.43 \\
\hline $\mathrm{FA}, \mathrm{n}(\%)$ & $2(3.30 \%)$ & $1(7.70 \%)$ & 0 & 0.57 \\
\hline Valvulopatía, n (\%) & $10(16.40 \%)$ & $1(7.70 \%)$ & 0 & 0.62 \\
\hline TAVI, n (\%) & $3(4.90 \%)$ & $1(7.70 \%)$ & 0 & 0.686 \\
\hline MITRACLIP, n (\%) & $2(3.30 \%)$ & 0 & 0 & 1 \\
\hline EAP, n (\%) & $1(1.60 \%)$ & $1(7.70 \%)$ & $1(14.30 \%)$ & 0.14 \\
\hline Cardiopatía congénita (\%) & $1(1.60 \%)$ & $1(7.70 \%)$ & 0 & 0.43 \\
\hline Miocardiopatía dilatada, n (\%) & $6(9.80 \%)$ & $1(7.70 \%)$ & 0 & 1 \\
\hline Dispositivo (\%) & $2(3.30 \%)$ & $2(15.40 \%)$ & 0 & 0.15 \\
\hline FEVI \% & $52(39.5-61)$ & $59(44-65)$ & $56(49-59)$ & 0.3 \\
\hline
\end{tabular}

Los datos se presentan en mediana y percentiles (p25-p75) o en $\mathrm{n}(\%)$. IMC: índice de masa corporal (kg/m²); DM2: diabetes mellitus tipo 2; HAS: hipertensión arterial sistémica; Cl: cardiopatía isquémica; IC: insuficiencia cardíaca; FA: fibrilación auricular; TAVI: implante valvular transaórtico; EAP: enfermedad arterial periférica; FEVI: fracción de expulsión del ventrículo izquierdo.

Los porcentajes de ganancias por percentil fueron los siguientes: para el grupo de MCT, 24.66 percentil $25^{\circ}, 45 \%$ percentil $50^{\circ}, 67.59 \%$ percentil $75^{\circ}$; para el grupo de EIMI, la ganancia en MET-carga fue de $26.77 \%$ percentil $25^{\circ}, 60 \%$ percentil $50^{\circ}$ y $94.84 \%$ percentil $75^{\circ}$; en el grupo de EIAI se identificó la mayor ganancia con $42.14 \%$ percentil $25^{\circ}, 85.74 \%$ percentil $50^{\circ}$ y $157.5 \%$ percentil $75^{\circ}$; se advierte una tendencia a mayor ganancia en el grupo EIAl, pero sin que sea estadísticamente significativa, $p=0.171$ (Fig. 2).

\section{Discusión}

Desde la década de 1960, los PRHCPS mediante la integración de actividades transdisciplinarias han tenido como objetivo mejorar la calidad de vida de los pacientes y lograr la reincorporación más completa posible a la sociedad ${ }^{8}$, y han ampliado su indicación a población con riesgo elevado de enfermedad cardiovascular, es decir, los individuos que tienen a la vez diversos factores de riesgo cardiovascular ${ }^{3}$.

La gradación del riesgo antes del inicio de un $\mathrm{PRH}$ CPS es una parte fundamental para la evaluación y prescripción de ejercicio en los individuos que ingresan a dichos programas; con base en lo anterior, diferentes asociaciones mundiales han categorizado a los pacientes en riesgo cardiovascular bajo, medio y alto mediante variables clínicas y prueba de esfuerzo convencional ${ }^{4}$, como la tolerancia al esfuerzo, presencia de umbral isquémico en la prueba de esfuerzo convencional, 
Tabla 3. Resultados de PRHCPS

\begin{tabular}{|l|c|c|c|c|}
\hline Variable & EIMC $\mathbf{n}=\mathbf{6 1}$ & EIMI $\mathbf{n}=13$ & EIAI $\mathbf{n}=\mathbf{7}$ \\
\hline BORG & $13(12-13)$ & $13(12-13.5)$ & $12(12-13)$ & 0.157 \\
\hline MET inicial & $6.2(4.6-7.2)$ & $4(3.05-6.2)$ & $4.1(2.5-11.3)$ & 0.15 \\
\hline MET final & $9(7.2-10.7)$ & $7.1(5.2-9.0)$ & $8.1(8-16.4)$ & 0.095 \\
\hline \% Ganancia MET & $45(24.66-67.59)$ & $60(26.77-94.84)$ & $85.74(42.14-157.5)$ & 0.171 \\
\hline$\%$ MET Máx inicial & $83(58.5-101.5)$ & $55(42-84)$ & $86(40-128)$ & 0.158 \\
\hline$\%$ MET Máx final & $115(94-138)$ & $89(76.5-122.5)$ & $127(72-182)$ & 0.216 \\
\hline Carga inicial (vatios) & $32(24-40)$ & $25.5(13-38.5)$ & $30(16-54)$ & 0.426 \\
\hline Carga final (vatios) & $50(37.75)$ & $42.5(31.5-57)$ & $42(39-75)$ & 0.390 \\
\hline \% Ganancia (vatios) & $51.51(25.83-84.29)$ & $44.29(30.5-81.93)$ & $48.64(34.32-118.75)$ & 0.544 \\
\hline
\end{tabular}

Los datos se presentan en mediana y percentiles (p25-p75\%) o en $n(\%)$. BORG: escala de percepción del esfuerzo, 6-12; MET: equivalente metabólico

(3.5 ml de $0, / \mathrm{kg} / \mathrm{min}) ; \%$ MET Máx inicial: porcentaje de MET-carga teóricos iniciales; \% MET Máx final: porcentaje de MET-carga teóricos finales; carga inicial en vatios; EIMC: entrenamiento moderado continuo; EIMI: entrenamiento interválico de moderada intensidad; ElAl: entrenamiento interválico de alta intensidad.

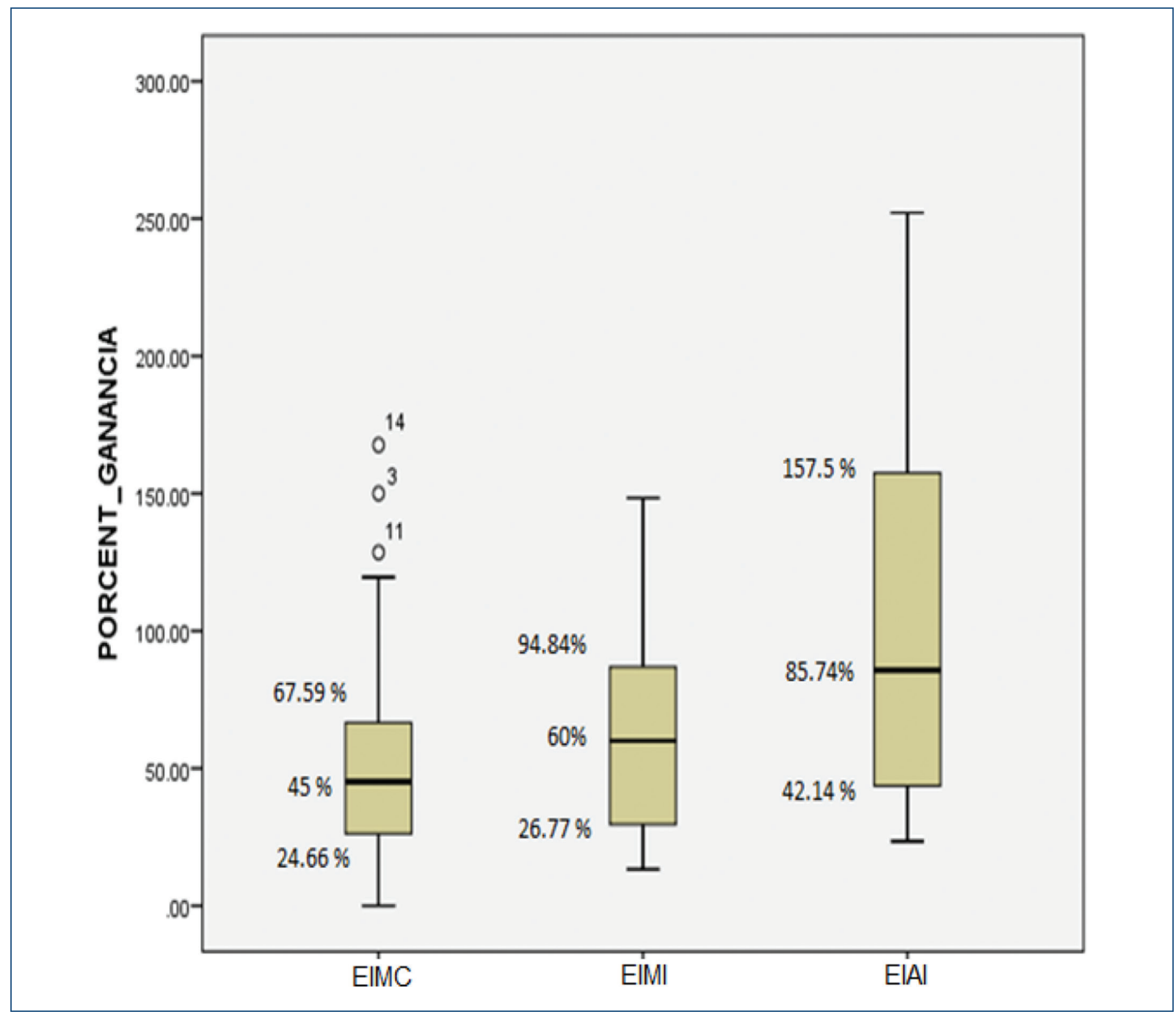

Figura 2. Porcentaje de ganancia en MET. Porcentaje de ganancias de $\mathrm{VO}_{2}$ en los tres grupos de intervención: $45 \%$ para EIMC, $60 \%$ para EIMI y $85.74 \%$ para EIAI, ( $p=0.17)$. EIMC: entrenamiento moderado continuo; EIMI: entrenamiento interválico de moderada intensidad; EIAI: entrenamiento interválico de alta intensidad. 
respuesta cronotrópica, recuperación de la frecuencia cardíaca al primer minuto, respuesta presora, recuperación de la tensión arterial sistólica y presencia de ectopia ventricular compleja (EVF) ${ }^{9-17}$. Sin embargo, en la realidad clínica un solo paciente puede albergar múltiples variables de alto riesgo cardiovascular. En este estudio, todos los pacientes que se evaluaron tuvieron más de un factor de riesgo cardiovascular, lo que los convierte en sujetos de riesgo cardiovascular alto, y por tanto pueden catalogarse de esa manera como de "riesgo cardiovascular muy alto". Este protocolo es el primero en evaluar el efecto del entrenamiento en EIMC, EIMI y EIAl en pacientes de muy alto riesgo cardiovascular.

El entrenamiento por intervalos consiste en una serie de episodios repetidos de intervalos de trabajo de mayor intensidad alternados con pausas de recuperación (ejercicios de intensidad ligera o leve $)^{18}$. La naturaleza intermitente del EIAI permite menos fatiga debido a los períodos de recuperación, lo que lleva a lograr una mayor intensidad de esfuerzo durante el intervalo. Como resultado, los sistemas de energía de trifosfato de adenosina-fosfocreatina (ATP-PC) y de glucólisis se usan una y otra vez, de tal manera que se promueve un aumento de la capacidad de energía de los músculos esqueléticos. Además, los múltiples períodos de recuperación hacen posible que el volumen sistólico alcance sus valores más altos varias veces durante un solo período de ejercicio en lugar de solo una vez con un período continuo de ejercicio ${ }^{18}$.

El entrenamiento en EIAI ha demostrado beneficios notables en la disminución de los factores de riesgo cardiovascular, mejoría del acondicionamiento físico y beneficios en la mortalidad por todas las causas. También se ha notificado que el EIAI es más efectivo que el EIMC en la reducción de grasa corporal debido a un gasto energético mayor durante el entrenamiento. Otras publicaciones han demostrado que las adaptaciones cardiovasculares que ocurren en EIAI son similares, e incluso superiores en algunos casos a las que se obtienen mediante EIMC ${ }^{19}$. Desde el decenio de 1980 se han investigado los beneficios del entrenamiento interválico de alta intensidad (EIAI) en pacientes con enfermedad arterial coronaria. En el metaanálisis de Martland, et al..$^{20}$ se demostró evidencia de que el EIAI mejora la función cardiorrespiratoria, composición corporal, valores de glucosa sérica, control glucémico, función endotelial y cardiovascular, frecuencia cardíaca, marcadores inflamatorios, tolerancia al esfuerzo, masa muscular, así como mejoría en estados de ansiedad y depresión ${ }^{20}$. Hasta el momento se han demostrado efectos positivos en ganancia de VO2 máximo, con algunos resultados contrastantes en pacientes de riesgo cardiovascular alto ${ }^{17,21-34}$. Sin embargo, prevalecen grandes interrogantes, por ejemplo ¿quién es el paciente elegible para realizar tratamiento interválico?, ¿en qué individuos no debe usarse? La mayoría de los estudios se ha realizado en personas relativamente jóvenes de género masculino, con función normal del ventrículo izquierdo, capacidad aeróbica > $7 \mathrm{MET}$, enfermedad coronaria estable y ausencia de signos 0 síntomas de isquemia miocárdica residual o arritmias ventriculares ${ }^{9,18}$.

Debido a que no todos los pacientes son aptos para EIAI, existe un modelo similar pero alternativo, esto es, el entrenamiento interválico de intensidad moderada (EIMI). Este método de entrenamiento se emplea a menudo al mismo volumen que el EIMC pero con pausas activas, tal y como se estructura en el EIAl. Se diferencia en que, en lugar de entrenar a los pacientes con intervalos de trabajo de 85 a $90 \%$ de FCR, se utiliza una cifra en cardiópatas de 70 a $80 \%$ de FCR y con dichas pausas entre 60 y $70 \%$ de $\mathrm{FCR}^{18}$. Keteyian, et al. ${ }^{35}$ describen tres efectos importantes con el tratamiento interválico: a) incorpora los efectos intermitentes y potencialmente más favorables del trabajo a intervalos sin que los pacientes excedan las pautas actuales para prescribir ejercicio; b) ayuda a los pacientes a trabajar en el extremo superior de su espectro de entrenamiento, lo que facilita la progresión del entrenamiento para generar desplazamiento de umbrales mayores, y c) favorece la adherencia al tratamiento, dado que los pacientes que usan EIMI o EIAI refieren que "disfrutan" mejor sus entrenamientos. Con frecuencia informan que obtienen un "mejor" entrenamiento con prácticas de intervalos, al margen de usar EIAI o EIMI. En los resultados de este trabajo, si bien no se evidencia una significancia estadística en las ganancias de tolerancia al esfuerzo evaluada por ganancia en MET, se advierte una tendencia mayor a la ganancia en porcentaje de MET inicial y final en los pacientes sometidos a la modalidad interválica, en particular con EIAI.

En las publicaciones médicas, al observar las ganancias en VO2p y el efecto que éstas tienen sobre la morbimortalidad, las investigaciones científicas han acumulado evidencia para trasladar ese efecto a las poblaciones con cardiopatía. No obstante, dado que se trata de intensidades de esfuerzo que pueden poner en riesgo a pacientes con estas características al someterlos por dos a cuatro minutos en inestabilidad metabólica, se ha logrado configurar este tratamiento por debajo del umbral ventilatorio 2 (máximo punto de 
eficiencia ventilatoria), con la finalidad de adaptarlos a estas ganancias y con el menor riesgo posible. Por esa razón, en cuanto a la seguridad de este protocolo, al igual que el metaanálisis de Hannan, et al. ${ }^{18}$, en el que se estudió la seguridad del entrenamiento interválico como EIMC, no se registraron episodios cardiovasculares que requirieran alguna hospitalización ni tampoco muertes. Hasta el momento, según lo demostrado en este estudio, estos resultados se han replicado con buen margen de seguridad, sin complicaciones adjuntas en los registros.

\section{Limitaciones}

Debido al principio de individualización del entrenamiento, en los pacientes que se integran al PRCPS no es posible establecer de manera aleatoria un tipo de entrenamiento; esto ha obligado a que la metodología carezca de aleatorización, con grupos controles basados en criterios de selección bajo normatividad ética en la prescripción y de acuerdo con la estratificación del riesgo cardiovascular. Si bien a todos los pacientes se les proporcionó entrenamiento concurrente, no existe algún método fidedigno para evaluar la magnitud del entrenamiento estático en la ganancia en tolerancia al esfuerzo. Se requiere una inclusión mayor de pacientes para reforzar los resultados de la muestra. Las mediciones se realizaron a través del VO2p calculado por carga y no a través de la norma de referencia, es decir, la medición con análisis de gases espirados.

\section{Conclusiones}

En cardiópatas de riesgo cardiovascular muy alto, el entrenamiento interválico EIMI/EIAI supervisado es seguro, con tendencia a una mayor ganancia en tolerancia al esfuerzo (MET-carga) con el EIAI en comparación con el EIMC.

\section{Financiamiento}

La presente investigación no ha recibido ninguna beca específica de agencias de los sectores público, comercial, o sin ánimo de lucro.

\section{Conflicto de intereses}

Los autores declaran que no tienen conflicto de intereses.

\section{Responsabilidades éticas}

Protección de personas y animales. Los autores declaran que para esta investigación no se han realizado experimentos en seres humanos ni en animales.

Confidencialidad de los datos. Los autores declaran que han seguido los protocolos de su centro de trabajo sobre la publicación de datos de pacientes.

Derecho a la privacidad y consentimiento informado. Los autores han obtenido el consentimiento informado de los pacientes o sujetos referidos en el artículo. Este documento obra en poder del autor de correspondencia.

\section{Bibliografía}

1. Wewege MA, Ahn D, Yu J, Liou K, Keech A. High-intensity interval training for patients with cardiovascular disease-is it safe? A systematic review. J Am Heart Assoc. 2018;7:e009305.

2. Gomes-Neto M, Durães AR, Conceição LSR, Saquetto MB, Ellingsen $\varnothing$, Carvalho VO. High intensity interval training versus moderate intensity continuous training on exercise capacity and quality of life in patients with heart failure with reduced ejection fraction: a systematic review and meta-analysis. International Journal of Cardiology. 261;134-141.

3. Maroto JM, Artigao-Ramírez R, Morales-Durán MD, de Pablo-Zarzosa C, Abraira V. Rehabilitación cardíaca en pacientes con infarto de miocardio. Resultados tras 10 años de seguimiento. Rev Esp Cardiol. 2005;58:1181-7.

4. França da Silva AK, da Costa de Rezende-Barbosa MP, Barbosa-Bernardo AF, Marques-Vanderlei F, Lopes-Pacagnelli F, Marques-Vandelei LC. Cardiac risk stratification in cardiac rehabilitation programs: a review of protocols. Rev Bras Cir Cardiovasc. 2014;29(2):255-65.

5. Quindry JC, Franklin BA, Chapman M, Humphrey R, Mathis S. Benefits and risks of high-intensity interval training in patients with coronary artery disease. Am J Cardiol. 2019;123(8):1370-1377.

6. Kim C, Choi HE, Lim YJ. The effect of cardiac rehabilitation exercise training on cardiopulmonary function in ischemic cardiomyopathy with reduced left ventricular ejection fraction. Ann Rehabil Med. 2016;40(4):647-656.

7. Lavie CJ, Ozemek C, Carbone S, Katzmarzyk PT1, Blair SN. Sedentary behavior, exercise, and cardiovascular health. Circ Res. 2019;124(5):799-815.

8. Mark DB, Hlatky MA, Harrell FE Jr, Lee KL, Califf RM, Pryor DB. Exercise treadmill score for predicting prognosis in coronary artery disease. Annals of Internal Medicine. 1987;106(6):793-800.

9. Dresing TJ, Blackstone EH, Pashkow FJ, Snader CE, Marwick TH, Lauer MS. Usefulness of impaired chronotropic response to exercise as a predictor of mortality, independent of the severity of coronary artery disease. Am J Cardiol. 2000;86(6):602-9.

10. Cole CR, Blackstone EH, Pashkow FJ, Snader CE, Lauer MS. Heart-rate recovery immediately after exercise as a predictor of mortality. $\mathrm{N}$ Engl J Med. 1999:341(18):1351-7.

11. Shetler K, Marcus R, Froelicher VF, Vora S, Kalisetti D, Prakash M, et al. Heart rate recovery: validation and methodologic issues. J Am Coll Cardiol. 2001;38(7):1980-7.

12. Myers J, Prakash M, Froelicher V, Do D, Partington S, Atwood JE. Exercise capacity and mortality among men referred for exercise testing. New England Journal of Medicine. 2002;346(11):793-801.

13. McHam SA, Marwick TH, Pashkow FJ, Lauer MS. Delayed systolic blood pressure recovery after graded exercise: an independent correlate of angiographic coronary disease. J Am Coll Cardiol. 1999;34(3):754-9.

14. Frolkis JP, Pothier CE, Blackstone EH, Lauer MS. Frequent ventricular ectopy after exercise as a predictor of death. N Engl J Med. 2003;348: 781-790.

15. Lauer MS, Okin PM, Larson MG, Evans JC, Levy D. Impaired heart rate response to graded exercise: prognostic implications of chronotropic Incompetence in the Framingham Heart Study. Circulation. 1996;93:15201526.

16. Morrow K, Morris CK, Froelicher VF, Hideg A, Hunter D, Johnson E, et al. Prediction of cardiovascular death in men undergoing noninvasive evaluation for coronary artery disease. Annals of Internal Medicine. 1993;118(9):689-695. 
17. Ilarraza-Lomelí H. Rehabilitación y prevención cardiovascular: el complemento necesario a la terapéutica de hoy. Arch Cardiol Mex. 2003;73: 247-252.

18. Hannan AL, Hing W, Simas V, Climstein M, Coombes JS, Jayasinghe R. High-intensity interval training versus moderate intensity continuous training within cardiac rehabilitation a systematic review and meta-analysis. Open Access Journal of Sports Medicine. 2018:9:1-17.

19. Milanović Z, Sporiš G, Weston M. Effectiveness of high-intensity interval training (hit) and continuous endurance training for VO2max improvements: a systematic review and meta-analysis of controlled trials. Sports Med. 2015;45(10):1469-81.

20. Martland R, Mondelli V, Gaughran F, Stubbs B. Can high-intensity interval training improve physical and mental health outcomes? A metareview of 33 systematic reviews across the lifespan. J Sports Sci. 2020; 38(4):430-469.

21. Nystoriak MA, Bhatnagar A. Cardiovascular effects and benefits of exercise. Front Cardiovasc Med. 2018;5(135):1-11.

22. Gibb AA, Epstein PN, Uchida S, Zheng Y, McNally LA, Obal D, et al. Exercise-induced changes in glucose metabolism promote physiological cardiac growth. Circulation. 2017;136:2144-57.

23. Linke A, Erbs S, Hambrecht R. Effects of exercise training upon endothelial function in patients with cardiovascular disease. Frontiers in Bioscience. 2008;13:424-432.

24. Hambrecht R, Wolf A, Gielen S, Linke A, Hofer J, Erbs S, et al. Effect of exercise on coronary endothelial function in patients with coronary artery disease. N Engl J Med. 2000;342(7):454-60

25. Ertek S, Cicero A. Impact of physical activity on inflammation: effects on cardiovascular disease risk and other inflammatory conditions. Arch Med Sci. 2012;8(5):794-804

26. Bousselmi R, Lebbi MA, Ferjani M. Myocardial ischemic conditioning: Physiological aspects and clinical applications in cardiac surgery. J Saudi Heart Assoc. 2014;26(2):93-100.
27. Winzer EB, Woitek F, Linke A. Physical activity in the prevention and treatment of coronary artery disease. J Am Heart Assoc. 2018;7(4):1-15.

28. Bruning RS, Sturek M. Benefits of exercise training on coronary blood flow in coronary artery disease patients. Prog Cardiovasc Dis. 2015;57(5):443-453.

29. Sandri M, Adams V, Gielen S, Linke A, Lenk K, Kränkel N, et al. Effects of exercise and ischemia on mobilization and functional activation of blood-derived progenitor cells in patients with ischemic syndromes: results of 3 randomized studies. Circulation. 2005;111(25):3391-3399.

30. Popovic D, Kumar N, Chaudhry S, Bagai A, Arena R, Kumar N. Improvements in key cardiopulmonary exercise testing variables following cardiac rehabilitation in patients with coronary artery disease. Cardiopulmonary Exercise Testing in Cardiac Rehabilitation. 2018;38(5):E5-E8.

31. Ribeiro F, Alves AJ, Teixeira M, Miranda F, Azevedo C, Duarte JA. Exercise training enhances autonomic function after acute myocardial infarction: a randomized controlled study. Rev Port Cardiol. 2012;31(2):135-141.

32. Hambrecht R, Niebauer J, Marburger C, Grunze M, Kälberer B, Hauer K, et al. Various intensities of leisure time physical activity in patients with coronary artery disease: effects on cardiorespiratory fitness and progression of coronary atherosclerotic lesions. J Am Coll Cardiol. 1993; 22(2):468-77.

33. Hambrecht R, Adams V, Erbs S, Linke A, Kränkel N, Shu Y, et al. Regular physical activity improves endothelial function in patients with coronary artery disease by increasing phosphorylation of endothelial nitric oxide synthase. Circulation. 2003;107(25):3152-8.

34. Badrov MB, Wood KN, Lalande S, Sawicki CP, Borrell LJ, Barron CC, et al. Effects of 6 months of exercise-based cardiac rehabilitation on autonomic function and neuro-cardiovascular stress reactivity in coronary artery disease patients. J Am Heart Assoc. 2019;8(17):1-10.

35. Keteyian SJ. High intensity interval training in patients with cardiovascular disease: a brief review of physiologic adaptations and suggestions for future research. Journal of Clinical Exercise Physiology. 2013;2(1):13-19. 